\title{
ON SOME CONJECTURES OF GRIGGS AND GRAFFITI
}

\author{
ERMELINDA DELAVINA, SIEMION FAJTLOWICZ, BILL WALLER
}

\begin{abstract}
We discuss a conjecture of J. R. Griggs relating the maximum number of leaves in a spanning tree of a simple, connected graph to the order and independence number of the graph. We prove a generalization of this conjecture made by the computer program Graffiti, and discuss other similar conjectures, including several generalizations of the theorem that the independence number of a simple, connected graph is not less than its radius.
\end{abstract}

\section{INTRODUCTION}

Graffiti, a computer program that makes conjectures, was written by S. Fajtlowicz. A later version of this program, called Dalmatian, was coauthored with E. DeLaVina. An annotated listing of several hundred of Graffiti's conjectures, dating from the program's inception in the mid-1980's, can be found in [8]. Graffiti has correctly conjectured a number of new bounds for several well-studied graph invariants; bibliographical information on resulting papers can be found in [3].

All graphs considered are simple and finite of order $n$. We let $\alpha=$ $\alpha(G)$ denote the independence number of a graph $G$, and $L=L(G)$ the maximum number of leaves over all spanning trees of the graph. Let $G$ be graph, and suppose $v$ is a vertex of $G$. Then the local independence number at $v$ is the independence number of the subgraph induced by the neighbors of vertex $v$. Let $\mu=\mu(G)$ be the maximum of the local independence numbers taken over all vertices of $G$. A subset of the vertices of $G$ that spans a connected subgraph and dominates the remaining vertices of $G$ is called a connected dominating set for $G$. The size of a smallest connected dominating set of $G$ is called the connected domination number of $G$. (Standard graph-theoretical terms not defined in this paper can be found in [16].)

Date: March 2002. Revised: May 2003.

1991 Mathematics Subject Classification. 05C35.

Key words and phrases. independence number, connected dominating set, radius, path covering number, Graffiti. 
In 1996, J. R. Griggs communicated the following conjecture to DeLaVina.

Conjecture 1. (Griggs) Let $G$ be a connected graph. Then

$$
L \geq n-2 \alpha+1 \text {. }
$$

Griggs further inquired as to whether Graffiti could make such a conjecture. DeLaVina's subsequent experiments with Graffiti resulted in the program conjecturing a number of other lower bounds for the invariant $L$. Many of these bounds are now known to be correct [2]. While determining $L$ exactly is NP-hard [12], quite a few papers have considered the problem of finding lower bounds for $L$ (see [10], [11] and [14]). Interestingly, Graffiti did not duplicate Griggs' conjecture. Instead, it discovered the following statement, which is stronger than Griggs' conjecture for graphs other than cliques.

Conjecture 2. (Graffiti) Let $G$ be a connected graph. Then

$$
L \geq n+\mu-2 \alpha-1 .
$$

A subset of vertices is a connected dominating set if and only if its complement is the set of leaves of a spanning tree. Therefore, inequalities of the form $L \geq n-i$, where $i$ is some graph invariant, are of interest because $i$ provides an upper bound on the connected domination number of $G$. These conjectures of Griggs and Graffiti state inequalities of this form. The connected domination number of a graph has been considered in several recent papers (see [1]).

DeLaVina and Fajtlowicz proved Griggs' conjecture shortly after Graffiti made the stronger conjecture. (Y. Caro communicated an independent proof to Griggs as well.) We defer all proofs until later in the paper; however, the proof of Conjecture 2 (likewise the original proof of Conjecture 1) is closely based on techniques used by Fajtlowicz and B. Waller in [9] to prove the following Theorem 1. This theorem results from one of Graffiti's earliest conjectures. Alternative proofs of this theorem are given by O. Favaron (see [8]) and Fajtlowicz (see [6]); the result also follows from a lemma due to F. Chung quoted in [5]. We let $r=r(G)$ denote the radius of a graph $G$.

Theorem 1. (Fajtlowicz and Waller) Let $G$ be a connected graph. Then

$$
\alpha \geq r
$$

The proof of Conjecture 2 can be extended to prove the following Theorem 2 due to Fajtlowicz. Note again that Theorem 2 is a strengthening of Theorem 1 for graphs other than cliques. As far as we are aware, it is the first known improvement of Theorem 1. 
Theorem 2. (Fajtlowicz) Let $G$ be a connected graph. Then

$$
\alpha \geq r+\mu-2 \text {. }
$$

For certain types of graphs, the inequality provided by Theorem 1 is sharp. For instance, paths and cycles of even order, or barbells with odd diameter, have this property. Thus Theorem 2 provides a necessary condition for this inequality to be sharp, namely, $\mu \leq 2$.

Fajtlowicz was inspired to suggest Theorem 2 because of another generalization of Theorem 1 made by Graffiti. A collection of vertex disjoint paths which cover all vertices of a graph $G$ is called a path covering of $G$. The size of a smallest path covering will be called the path covering number of $G$; we use $\rho=\rho(G)$ to denote this number. In [13], L. Lovasz found a simple, but nice bound for the independence number: $\alpha \geq \rho$. In light of this bound and Graffiti's bound $\alpha \geq r$, the following of Graffiti's conjectures was of particular interest to us.

Conjecture 3. (Graffiti [7]) Let $G$ be a connected graph. Then

$$
\alpha \geq r+\rho-1 \text {. }
$$

The " -1 " term is required, for otherwise cliques with more than one vertex would be obvious counterexamples. We note that the conjecture is true for values of $r \leq 3$; Lovasz proved this within hours of learning of the conjecture [private communication]. However, DeLaVina and Waller have found counterexamples to this conjecture for all $r \geq 4$ [4]. In fact, they demonstrate a family of trees $\left\{T_{k} \mid k=1,2,3, \ldots\right\}$ where

$$
r\left(T_{2 k}\right)+\rho\left(T_{2 k}\right)-\alpha\left(T_{2 k}\right)=k .
$$

For the sake of completeness, we will repeat the construction of $T_{2 k}$ in the next section. Upon being informed of counterexamples to Conjecture 3, Graffiti made the following two conjectures.

Conjecture 4. (Graffiti) Let $G$ be a connected graph. Then

$$
\alpha \geq\left\lfloor\frac{r}{2}\right\rfloor+\rho
$$

Conjecture 5. (Graffiti) Let $G$ be a connected graph. Then

$$
\alpha \geq r+\frac{\rho-1}{2} .
$$

Figure 1 shows an example of equality for Conjectures 4 and 5 . They remain open in the general case. However, both conjectures are true if restated for trees, as shown by the following two theorems. The proof of Theorem 3 is derived from the counterexamples to Conjecture 4 mentioned earlier and is given in [4]; the proof of Theorem 4 is given in the next section. In section 3 , we list some additional conjectures 


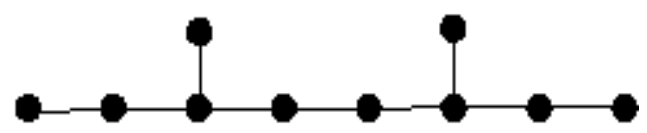

FiguRE 1. Example of equality for Conjectures 4 and 5

regarding the independence number that Graffiti generated at the same time as Conjectures 4 and 5 .

Theorem 3. (DeLaVina and Waller) Let $T$ be a tree of order more than 2 and suppose $d$ is the number of vertices contained on a path in $T$ of maximum length (i.e. $d$ is one more than the diameter of $T$ ). Put $x=\lfloor d / 3\rfloor$. If $x$ is even, then

$$
\alpha \geq\left(\frac{2 x}{3 x+2}\right) r+\rho .
$$

On the other hand, suppose $x$ is odd. Then

$$
\alpha \geq\left(\frac{2 x}{3 x+1}\right) r+\rho .
$$

Moreover, both bounds are sharp, for all possible values of $x$.

Theorem 4. Let $T$ be a tree. Then

$$
\alpha \geq r+\frac{\rho-1}{2} .
$$

Of course, this bound is sharp for paths of even order. The tree in Figure 1 shows this bound is sharp, even when $\rho>1$. Furthermore, one can easily extend this tree to demonstrate other cases of equality where $\rho>1$, for all $r \geq 4$.

\section{Proofs of Main Results}

Proof of Conjecture 2. In a 1986 paper by Fajtlowicz and Waller [9], a connected dominating set was called a trunk, since any trunk for $G$ can be used to create a spanning tree where each of the non-trunk vertices of $G$ is a leaf of the spanning tree. Now in trying to establish inequalities of the form $L \geq n-i$, where $i$ is some graph invariant, it is enough to show that $G$ has a connected dominating set with no more than $i$ vertices. Thus in order to prove Conjecture 2, we need only show the existence of a connected dominating set of size at most $2 \alpha-\mu+1$. Lemma 1 provides such a set. This lemma is essentially Waller's Lemma 
5.2 given in [15]; however, for the purposes of this paper we begin with a vertex that realizes maximum local independence.

Lemma 1. Suppose $G$ is a connected graph such that $\mu \geq 2$ (i.e. $G$ is not a clique). Then there exists a maximal independent subset $M$ of the vertices of $G$, and a connected dominating set $T$ containing $M$, such that $T$ has no more than $2|M|-\mu+1$ vertices.

Proof. Let $c$ be a vertex such that the local independence number at $c$ is $\mu$, and let $S$ be a maximum independent subset of vertices of the subgraph spanned by $c$ and its neighbors. Since $\mu \geq 2$, it follows $c \notin S$. We will inductively construct a sequence $T_{1}, T_{2}, \ldots, T_{k}$ of subsets of vertices of $G$ where each subset spans a connected subgraph and $T_{k}$ is a connected dominating set. In addition, we will define sequences $M_{1}, M_{2}, \ldots, M_{k} ; B_{0}, B_{1}, \ldots, B_{k-1} ;$ and $N_{1}, N_{2}, \ldots, N_{k}$ of subsets of vertices of $G$ such that for $j=1,2, \ldots, k$ :

1) $M_{j}$ is independent and $\left|M_{j}\right|=\mu+j-1$,

2) $\left|B_{j-1}\right| \leq j$,

3) $N_{j}$ is the set of all neighbors of vertices in $M_{j}$,

4) $B_{j-1} \subset N_{j}$, and

5) $B_{j-1} \cap M_{j}=\emptyset$ and $T_{j}=B_{j-1} \cup M_{j}$.

We begin by taking $M_{1}=S, B_{0}=\{c\}$, and $T_{1}=M_{1} \cup B_{0}$. Clearly $T_{1}$ spans a connected subgraph and Conditions $1-5$ are satisfied.

Now suppose each of the sets $T_{j}, M_{j}, B_{j-1}$, and $N_{j}$ have been defined for $j \geq 1$ and satisfy the given conditions. If $M_{j}$ is a maximal independent set, then $T_{j}$ must be a connected dominating set, so we put $M=M_{j}$ and $T=T_{j}$ and stop. Since,

$\left|T_{j}\right|=\left|B_{j-1} \cup M_{j}\right|=\left|B_{j-1}\right|+\left|M_{j}\right| \leq \mu+2 j-1=2|M|-\mu+1$, we are finished.

If $M_{j}$ is not maximal, then because $G$ is connected, there exists a vertex $v \notin M_{j} \cup N_{j}$, and a vertex $u \in N_{j}$, such that $v$ is adjacent to $u$. Put $M_{j+1}=M_{j} \cup\{v\}, B_{j}=B_{j-1} \cup\{u\}$, and $T_{j+1}=T_{j} \cup\{v, u\}$. Then again, clearly $T_{j+1}$ spans a connected subgraph and Conditions 1-5 are satisfied. The lemma now follows by induction.

Proof of Theorem 2. (DeLaVina and Fajtlowicz) We can assume G is not a clique. Apply the algorithm described in the proof of Lemma 1 to $G$. Clearly the diameter of the subgraph spanned by $T_{1}$ is 2 . Let $G_{j}$ be a tree spanned by the vertices of $T_{j}$ for $j=1,2, \ldots, k$. Then for $j<k$, one can assume the diameter of $G_{j+1}$ is at most two more than the diameter of $G_{j}$. Hence, one can moreover assume the diameter of $G_{k}$ is at most $2 k$, and consequently, the radius of $G_{k}$ is at most $k$. But recall $T=T_{k}$ is a dominating set for $G$, therefore the radius of $G$ 


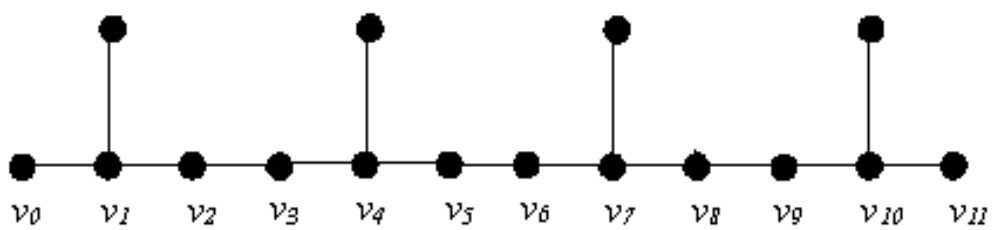

Figure 2. $T_{4}$, a counterexample to Conjecture 3

is at most $k+1$. Finally, recall $M=M_{k}$ is an independent set and $\left|M_{k}\right|=\mu+k-1$. Thus, $\alpha \geq|M|=\mu+k-1 \geq \mu+r-2$.

Counterexamples to Conjecture 3. (DeLaVina and Waller) Consider a path $P_{3 k}$ with $3 k$ vertices. Enumerate the vertices of $P_{3 k}$ from left to right as $v_{0}, v_{1}, v_{2}, \ldots, v_{3 k-1}$. Let $T_{k}$ be the tree on $4 k$ vertices formed by attaching a single edge to $P_{3 k}$ at each of the vertices $v_{j}$ where $j=1$ ( $\bmod 3)$. Thus $T_{1}$ is a star with 3 endpoints; $T_{2}$ is formed by taking two copies of $T_{1}$ and adding a single edge from an endpoint of one of the stars to an endpoint of the other; and so forth. Figure 2 illustrates $T_{4}$. It is easy to convince oneself, by inspection, that

$$
r\left(T_{4}\right)+\rho\left(T_{4}\right)-\alpha\left(T_{4}\right)=2 .
$$

In general, though, in [4] it is shown that for the tree $T_{2 k}$,

$$
r\left(T_{2 k}\right)+\rho\left(T_{2 k}\right)-\alpha\left(T_{2 k}\right)=k .
$$

Proof of Theorem 4. Let $P$ be a diametric path in $T$. Then we can choose two disjoint independent sets $A$ and $B$ from $P$ whose size meets or exceeds the radius of $T$. Let $F$ be the forest formed by deleting $P$ from $T$. If $F$ is empty, then $\rho=1$ and we are done. So suppose otherwise. Let $L$ be a minimum path covering of $F$, and let $S$ be a set formed by choosing one endpoint from each path in $L$. Thus $S$ must be an independent set in $T$, for if not, then $L$ is not minimum. Note also $\rho \leq|S|+1$. Let $M$ be the vertices in $S$ adjacent to vertices in $A$ (with respect to $T$ ), and likewise let $N$ be the vertices in $S$ adjacent to vertices in $B$. Clearly $M$ an $N$ are disjoint. Assume $|M| \geq|N|$. Then $|S-N| \geq|N|$. Note also $B \cup(S-N)$ is an independent set in $T$. But, 


$$
\begin{aligned}
\alpha & \geq|B \cup(S-N)|=|B|+|S-N| \\
& \geq r+\frac{|S-N|+|S-N|+1-1}{2} \\
& \geq r+\frac{|S-N|+|N|+1-1}{2} \\
& =r+\frac{|S|+1-1}{2} \\
& \geq r+\frac{\rho-1}{2} .
\end{aligned}
$$

\section{Other Conjectures of Graffiti}

Graffiti generated numerous other conjectures regarding the independence number at the same time it generated Conjectures 4 and 5 . The conjectures listed here are a few of the more interesting or simply stated of those.

Conjecture 6. (Graffiti) Let $G$ be a graph. Then

$$
\alpha \geq r+\ln (\rho) \text {. }
$$

Conjecture 7. (Graffiti) Let $G$ be a graph. Then

$$
\alpha \geq \ln (r)+\rho .
$$

Conjecture 8. (Graffiti) Let $G$ be a graph. Then

$$
\alpha \geq \ln \text { (chromatic number of the complement). }
$$

Although the previous conjecture is false, it is of interest because it suggests the classical problem of finding a minimal graph with (large) chromatic number $\chi$ and (small) clique number $\omega$. (See, for instance, [16].) The complement of such a graph would be a counterexample.

Acknowledgments: The authors wish to thank the referee for comments regarding the origin of Conjecture 1; and L. Lovasz for permission to quote his result regarding Conjecture 3. 


\section{REFERENCES}

[1] Yair Caro, Douglas B. West and Raphael Yuster, Connected Domination and Spanning Trees with Many Leaves, SIAM J. Disc. Math., 13(2000), p. 202-211.

[2] Ermelinda DeLaVina, Written on the Wall II, Web address: http://cms.dt.uh.edu/faculty/delavinae/wowII.ps

[3] Ermelinda DeLaVina, Web site of bibliographical information on conjectures of Graffiti, Web address: http://cms.dt.uh.edu/faculty/delavinae/wowref.html

[4] Ermelinda DeLaVina and Bill Waller, Independence, Radius and Path Coverings in Trees, Congressus Numerantium, 156(2002), p. 155-169.

[5] Paul Erdos, Michael Saks and Vera Sos, Maximum Induced Trees in Graphs, Journal of Graph Theory, 41(1986), p. 61-79.

[6] Siemion Fajtlowicz, A Characterization of Radius-Critical Graphs, Journal of Graph Theory, 12(1988), p. 529-532.

[7] Siemion Fajtlowicz, http://mathforum.org/epigone/sci.math.research/ delbrulha/8cbh7p\$ef0@Bayou.UH.EDU, April 2000.

[8] Siemion Fajtlowicz, Written on the Wall, Web address: http://math.uh.edu/ siemion

[9] Siemion Fajtlowicz and William Waller, On Two Conjectures of Graffiti, Congressus Numerantium, 55(1986), p. 51-56.

[10] Jerrold R. Griggs and Mingshen Wu, Spanning Trees in Graphs of Minimum Degree 4 or 5, Discrete Math, 104(1992), p. 167-183.

[11] Jerrold R. Griggs, Daniel J. Kleitman and Aditya Shastri, Spanning Trees with Many Leaves in Cubic Graphs, Journal of Graph Theory, 13(1989), p. 669-695.

[12] Paul Lemke, The Maximum Leaf Spanning Tree Problem for Cubic Graphs is NP-complete, preprint, The Institute for Mathematics and its Applications, 1988.

[13] Lazlo Lovasz, Combinatorial Problems and Exercises, Academiai Kiado, 1979, p. 55 .

[14] Guoli Ding, Thor Johnson and Paul Seymour, Spanning Trees with Many Leaves, Journal of Graph Theory, 37(2001), p. 189-197.

[15] William Waller, Average Distance in Graphs with Prescribed Order and Independence Number, Ph.D. Dissertation (1989), University of Houston, Houston, TX 77204, p. 35.

[16] Douglas B. West, Introduction to Graph Theory (2nd. ed.), Prentice-Hall, 2001.

DeLaVina and Waller, University of Houston-Downtown

E-mail address: delavinae@uhd.edu

FAJTlOWICZ, University OF Houston

E-mail address: matho@bayou.uh.edu 